\title{
Coordinated Voltage Control of Active Distribution Network
}

\author{
Jiang Xie ${ }^{1, a}$, Wenxia Liu ${ }^{1}$ and Huiting $\mathrm{Xu}^{1}, \mathrm{Xu} \mathrm{Han}^{2}$,Shuya Niu ${ }^{1}$ \\ ${ }^{1}$ School of Electrical and Electronic Engineering, North China Electric Power University, 102206 Beijing, China \\ 2 State Grid Baicheng Power Supply Company, 137000 Baicheng, China
}

\begin{abstract}
This paper presents a centralized coordinated voltage control method for active distribution network to solve off-limit problem of voltage after incorporation of distributed generation (DG). The proposed method consists of two parts, it coordinated primal-dual interior point method-based voltage regulation schemes of DG reactive powers and capacitors with centralized on-load tap changer (OLTC) controlling method which utilizes system's maximum and minimum voltages, to improve the qualified rate of voltage and reduce the operation numbers of OLTC. The proposed coordination has considered the cost of capacitors. The method is tested using a radial edited IEEE-33 nodes distribution network which is modelled using MATLAB.
\end{abstract}

\section{Introduction}

Large-scale development of DG, will bring problem to traditional distribution network such as bidirectional power flow and voltage off-limit. Traditional distribution network is designed based on the assumption that power flow is unidirectional and the network does not contain any controlled resources. Voltage control is mainly through OLTC and shunt capacitors in substation. These devices are run locally in most distribution network, without any communication or coordination with each other [1] [2]. In [3], the author proposed a simple automatic voltage control method, which controls local reactive power output of DG, reducing active power output of DG as well. These local control are usually restricted to one bus voltage, relying only on local measurements.

In fact, information and controllable equipment of all network can be used basing on more and more convenient measurement and communication system. In [4], the author has proposed a coordinated voltage control method of substation OLTC and reactive output of DERs based on maximum and minimum voltage of the network. As the number of controllable components increase, the difficulty of determining control rules becomes increasingly complex. In this paper, a centralized coordinated voltage method has been proposed including coordination between primal-dual interior point method-based voltage regulation schemes of DG reactive powers and capacitors, and centralized on-load tap changer (OLTC) controlling method which utilizes the system's maximum and minimum voltages, to ensure a stable and economical distribution network voltage.

\section{Proposed centralized coordinated voltage control}

\footnotetext{
a Jiang Xie : xiejiang12334@163.com

(C) The Authors, published by EDP Sciences. This is an open access article distributed under the terms of the Creative Commons Attribution License 4.0 (http://creativecommons.org/licenses/by/4.0/).
} 
The proposed method consists of two parts: primal-dual interior point method-based voltage regulation schemes of DG reactive powers and capacitors, centralized on-load tap changer (OLTC) controlling method which utilizes the system's maximum and minimum voltages proposed in [5].

\subsection{Model designs for optimizing schemes}

\subsubsection{Objective function}

$$
M \text { in } I=I_{v}+I_{Q D G}+I_{C}
$$

Objective function consists of three parts,

1) Minimizing extreme value of voltage

Coordination between optimizing schemes and centralized OLTC control is also reflected by this sub-objective function:

$$
I_{v}=\omega_{v} \frac{\Delta V_{\text {Upper }}+\Delta V_{\text {Lower }}}{\Delta V_{\max }}
$$

where

$$
\begin{aligned}
& \Delta V_{\text {Upper }}= \begin{cases}V_{\max }^{\text {system }}-V_{\text {Upper }} & V_{\max }^{\text {system }}>V_{\text {Upper }}, \\
0 & V_{\max }^{\text {system }} \leq V_{\text {Upper }}\end{cases} \\
& \Delta V_{\text {Lower }}= \begin{cases}V_{\text {Lower }}-V_{\min }^{\text {ssstem }} & V_{\min }^{\text {system }}<V_{\text {Lower }} \\
0 & V_{\min }^{\text {system }} \geq V_{\text {Lower }}\end{cases} \\
& \Delta V_{\max }=V_{\text {Upper }}-V_{\text {Lower }}
\end{aligned}
$$

where $\omega_{v}$ is proportionality coefficient; $V_{\text {Upper }}$ and $V_{\text {Lower }}$ respectively are standard upper and lower limit of feeder voltage, eg 1.07 p.u. and 0.93 p.u.; $V_{\min }^{\text {ssstem }}$ and $V_{\min }^{\text {ssstem }}$ respectively are maximum and minimum voltage of the network measured.

2) Minimizing use amount of reactive power of DGs

DGs' reactive power are finite:

$$
I_{Q D G}=\omega_{Q D G} \frac{\sum_{i=1}^{N_{D G}}\left|Q_{D G, i}\right|}{\sum_{i=1}^{N_{D G}}\left|Q_{D G, i}^{\max }\right|}
$$

where $\omega_{Q D G}$ is proportionality coefficient; $N_{D G}$ is total number of DGs; $Q_{D G i}$ is reactive power of DG i; $Q_{D G i}^{\max }$ is maximal reactive power of DG $\mathrm{i}$, and $Q_{D G i}^{p f}=P_{D G i} \tan \left(\cos ^{-1}\left(p f_{D G i}\right)\right), Q_{D G i}^{S}=\sqrt{S_{D G i}^{2}-P_{D G i}^{2}}$,

$Q_{D G i}^{v}=\sqrt{\left(\frac{V_{D G i}^{\max } V_{g}}{X}\right)^{2}-P_{D G i}^{2}}-\frac{V_{g}^{2}}{X}$, where $P_{D G i}$ is active power of DG; $V_{D G i}^{\max }$ is maximal output voltage of DG; $p f_{D G i}$ is power factor of DG; $S_{D G i}$ is apparent power of DG; $V_{g}$ is voltage of system power supply; X represents the total reactance of the DG interfacing transformer and filter in case of inverter-based DG or it represents the synchronous reactance in case of synchronous machine-based DG. For inverterbased DG, $V_{D G i}^{\max }$ relies on the dc-link voltage, while for synchronous machine-based DG $V_{D G i}^{\max }$ depends on the field voltage.

3) Minimizing the cost of using shunt capacitors 
Limiting the frequency of switching capacitor is intended to ensure the normal life of the compensating device and reduce the workload of operation and maintenance:

$$
I_{C}=\omega_{q} \sum_{i=1}^{C_{N}} \Delta q_{j}
$$

where $C_{N}$ is the total number of nodes with shunt capacitor bank; $\omega_{q}$ is the cost factor of each switching operation of capacitor; $\Delta q_{j}$ is the change in the number of compensation capacitor of node $\mathrm{j}$ before and after each optimization. In general, $\omega_{v}$ and $\omega_{Q D G}$ are all set to 1 .

\subsubsection{Constraints}

1) Power flow constraints

$$
G(x, u)=0
$$

where $\mathrm{x}$ and $\mathrm{u}$ respectively is state variable and control variable.

2) Constrains of reactive power of DGs

$$
\left|Q_{D G i}\right| \leq Q_{D G i}^{\max }
$$

where $Q_{D G i}$ is reactive power of DG i; $Q_{D G i}^{\max }$ is maximal reactive power of DG i.

3) Constrains of capacitors

$$
0 \leq q_{j} \leq q_{j, \max } \quad\left(j=1,2, \cdots, C_{N}\right)
$$

where $q_{j}$ is compensation capacity of shunt capacitor bank in node $\mathrm{j} ; q_{j, \max }$ is total capacity of shunt capacitor bank in node $\mathrm{j} ; c_{N}$ is the total number of nodes with shunt capacitor bank.

4) Constrains of voltage magnitude

The node voltage amplitude must be controlled at rated voltage of $7 \% \sim 7 \%$.

From a mathematical point of view, optimizing schemes are multi-objective nonlinear programming, and mixed-integer programming.

\subsection{Algorithm}

Primal-dual interior point method is used to deal with mixed integer optimization. Continuous control variables are reactive power output of DGs, discrete control variables is tap position of OLTC and numbers of groups of shunt capacitor banks switching on.

\subsection{Evaluation Index}

Comprehensive evaluation index is used to evaluate the proposed control strategy:

$$
S I=\frac{1}{m \times n} \sum_{i=1}^{m} \sum_{j=1}^{n}\left|\Delta U_{i j}\right|
$$

For further analysis and comparison, average offset dimension of voltage of each sample point is: 


$$
A V O_{i}=\frac{1}{n} \sum_{j=1}^{n}\left|\Delta U_{i j}\right|
$$

where $\mathrm{i}$ is sampling point, $\mathrm{j}$ is node number, and $\mathrm{m}$ is total number of sampling point set as 96 for 15 minutes interval between $\mathrm{i}$ and $\mathrm{i}+1, \mathrm{n}$ is total number of nodes, $\left|\Delta U_{i j}\right|$ is absolute value of offset dimension of voltage of node $\mathrm{j}$ at sampling point $\mathrm{i}$.

\section{Simulation result}

The simulation takes modified IEEE-33 system. Rated voltage is $12.66 \mathrm{kV}$. Intermittent solar energy can significantly change the system voltage profile and is usually located at the end of feeder. So 4 solar-based DGs are connected at different positions. The total number of tap positions of substation OLTC is 19. The time for voltage control is a typical 24-hour day, control interval is 15 minutes, and the total number of sampling is 96.The topological graph of IEEE-33 system is shown in figure 1. Curves of power of residential loads and solar-based DG during a day are shown in figure 2 . Node 2 , 15,31 respectively are installed with shunt capacitor bank, each group of capacity is $200 \mathrm{kvar}$, and number of groups are 10,3, and 3, respectively.

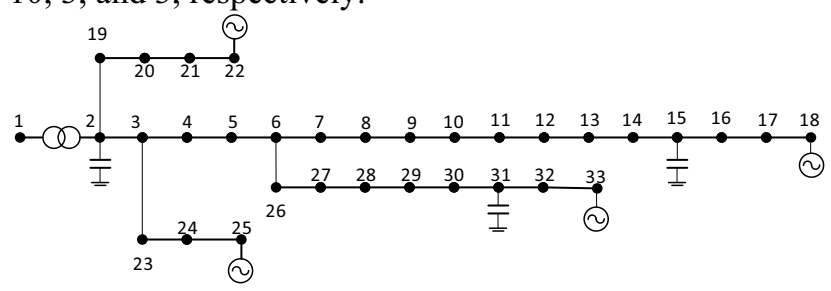

Figure 1. Modified IEEE-33 power system.

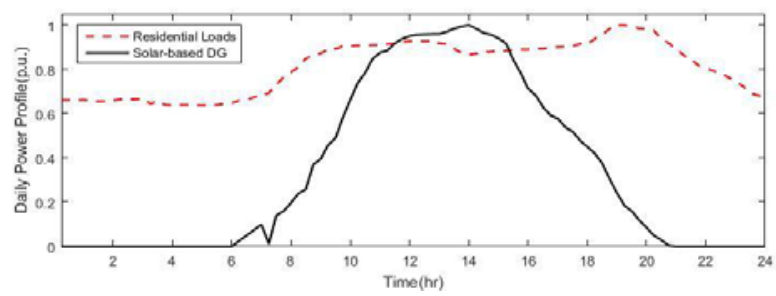

Figure 2. Power of residential loads and solar-based DG

The capacity of four DG is 3 MVA. Range of power factor are set to 0.95 lagging to 0.95 leading. To easily test effect of proposed coordinated voltage control scheme, assume the distributed photovoltaic DGs adopt decoupling control of active and reactive power. Figure 3 shows system's maximum and minimum voltages under two cases:

Case 1: Power factors of all DGs are unit, numbers of all shunt capacitor bank are zero. OLTC, shunt capacitor banks and reactive power of DGs are not controlled.

Case 2: Tap position, shunt capacitor bank and reactive power of DGs is all controlled using proposed coordinated voltage control scheme. Active power of DGs output is same as case 1 . On the other hand, reactive power of DGs is adjustable according to constraints shown in formula (6).

Shown in figure 3 and 4, system's maximum voltage is off-limit of 1.07 p.u. during about 12:00 to 16:00 when DGs have greater active power output, system's minimum voltage is off-limit of 0.93 p.u. during about 20:00 to 22:00 without any control; Proposed coordinated control scheme have solved the off-limit problem of voltage successfully, remarkably reduced average offset dimension of voltage specially in both overvoltage and under-voltage. 


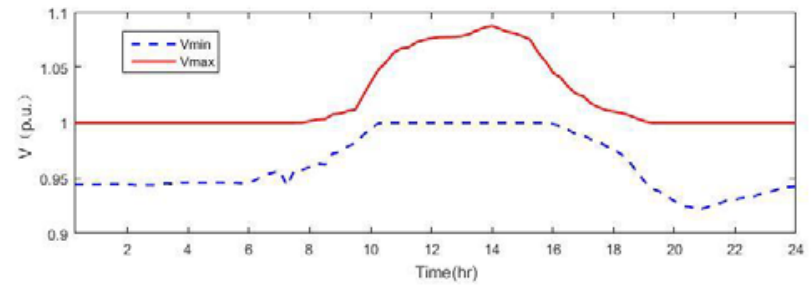

(a)

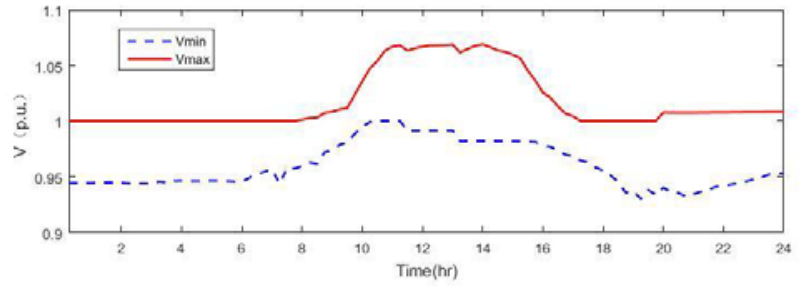

(b)

Figure 3. System's maximum and minimum voltages; (a) Without control, (b) Proposed coordinated control

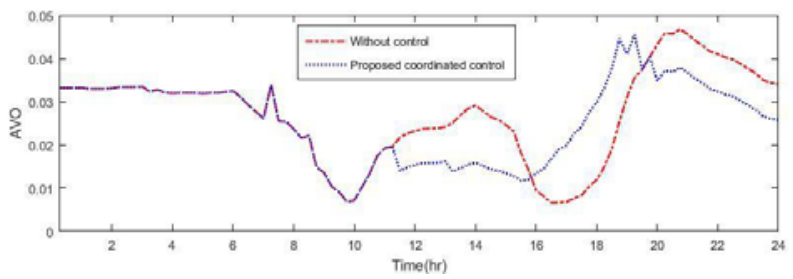

Figure 4. Average offset dimension of voltage (AVO)

Proposed method is contrasted by centralized voltage control method without capacitors. Proposed centralized coordinated voltage control scheme has considered the effect of shunt capacitor banks because shunt reactive power compensation can help improve under-voltage. Figure 5 shows the tap position of both scheme. The proposed scheme has decreased daily tap operation from 9 to 7 taps/day. Comprehensive evaluation index of all three cases respectively are $0.0270,0.0260$ and 0.0257 shown in table 1, both centralized and proposed centralized coordinated voltage control scheme can reduce offset dimension of system voltage, effect of proposed method this paper is better specially in undervoltage result from heavy load and light DG output.

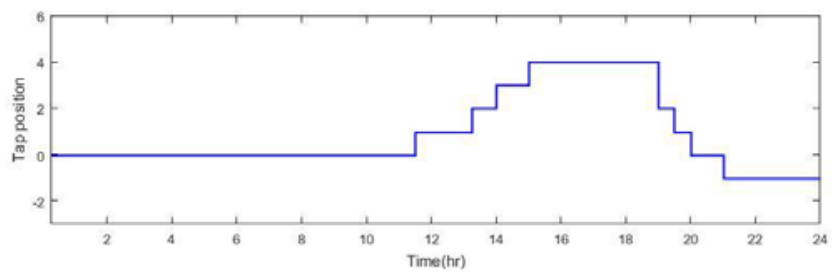

(a)

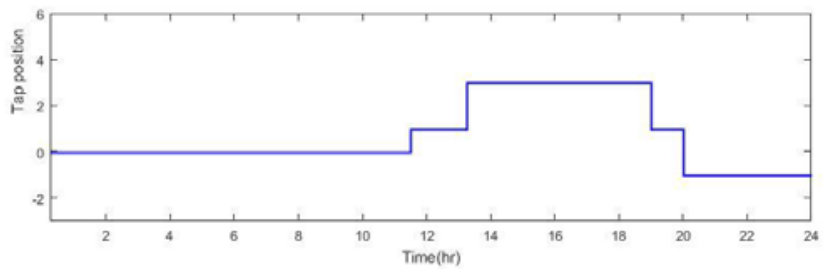


(b)

Figure 5.Tap position of OLTC; (a) Control without capacitors, (b) Proposed coordinated control.

Table 1. Comprehensive evaluation index (SI).

\begin{tabular}{|c|c|c|c|}
\hline Element & Without control & $\begin{array}{c}\text { Centralized control without } \\
\text { capacitors }\end{array}$ & Proposed coordinated control \\
\hline SI & 0.0270 & 0.0260 & 0.0257 \\
\hline
\end{tabular}

\section{Conclusion}

This paper presents a centralized coordinated voltage control scheme suited for active power distribution network. Once appears off-limit voltage, the reactive power of DG and shunt capacitor bank is optimized using primal-dual interior point method first of all, coordinated with substation OLTC by setting specific objective function then, to provide a more relaxed operation of the OLTC which can increase its life time; Especially in the optimization part, the proposed coordination has considered the cost of capacitor and incorporated it in whole coordinated control, reducing voltage excursion of active distribution network and decreased operation of OLTC facing heavy loading.

\section{References}

1. T.A.Short,Electric power distribution handbook Boca Raton,FL: CRC,(2004)

2. T.Gonen,Electric power distribution system New York: McGraw,(1986)

3. W.Freitas,J.C.M Vieira,A.Morelato,W.Xu,Influence of excitation system control modes on the allowable penetration level of distributed synchronous generators, IEEE Transactions on Energy Convers,2005,20(2): $474-480$

4. C.M.Hird,H.Leite,N.Jenkins,H.Li.Network voltage controller for distributed generation,IEEE Transactions on Generation,2004,151(2): 150 - 156

5. Maher A. Azzouz and Ehab F. El-Saadany. Optimal coordinated Volt/Var control in active distribution networks[C]// PES General Meeting, 2014 IEEE. IEEE, 2014:1-5 